\title{
The Progenetix oncogenomic resource in 2021
}

Qingyao Huang ${ }^{1,2}$, Paula Carrio-Cordo ${ }^{1,2}$, Bo Gao ${ }^{1,2}$, Rahel Paloots ${ }^{1,2}$, Michael Baudis $^{1,2 \propto, \dagger}$

1 Institute of Molecular Life Sciences, University of Zurich, Zurich, Switzerland 2 Swiss Institute of Bioinformatics, Zurich, Switzerland

aCurrent Address: Institute of Molecular Life Sciences, University of Zurich, Winterthurerstr. 190, CH-8057 Zürich

$\dagger$ michael.baudis@mls.uzh.ch

\section{Abstract}

In cancer, copy number aberrations (CNA) represent a type of nearly ubiquitous and frequently extensive structural genome variations. To disentangle the molecular mechanisms underlying tumorigenesis as well as identify and characterize molecular subtypes, the comparative and meta-analysis of large genomic variant collections can be of immense importance. Over the last decades, cancer genomic profiling projects have resulted in a large amount of somatic genome variation profiles, however segregated in a multitude of individual studies and datasets. The Progenetix project, initiated in 2001, curates individual cancer CNA profiles and associated metadata from published oncogenomic studies and data repositories with the aim to empower integrative analyses spanning all different cancer biologies.

During the last few years, the fields of genomics and cancer research have seen significant advancement in terms of molecular genetics technology, disease concepts, data standard harmonization as well as data availability, in an increasingly structured and systematic manner. For the Progenetix resource, continuous data integration, curation and maintenance have resulted in the most comprehensive representation of cancer genome CNA profiling data with 138'663 (including 115'357 tumor) CNV 
profiles. In this article, we report a 4.5-fold increase in sample number since 2013, improvements in data quality, ontology representation with a CNV landscape summary over 51 distinctive NCIt cancer terms as well as updates in database schemas, and data access including new web front-end and programmatic data access. Database URL: progenetix.org

\section{Introduction}

Copy number aberrations (CNA) are present in the majority of cancer types and exert functional impact in cancer development [1, 2]. As understanding cancer biologies remains one of the main challenges in contemporary medical and life sciences, the number of studies addressing genomic alterations in malignant diseases continues to grow. Progenetix is a publicly accessible cancer genome data resource (progenetix.org) that aims to provide a comprehensive representation of genomic variation profiles in cancer, through providing sample-specific CNA profiles and associated metadata as well as services related to data annotation, meta-analysis and visualization. Originally established in 2001 with a focus on data from chromosomal Comparative Genomic Hybridization (CGH) studies [3], the resource has progressively incorporated data from hundreds of publications reporting on molecular-cytogenetic (CGH, genomic arrays) and sequencing (whole genome or whole exome sequencing; WGS, WES) based genome profiling experiments. Since the last publication dedicated to the Progenetix resource in 2014 [4], changes in content and features of the data repository and its online environment have vastly expanded its scope and utility to the cancer genomics community. For data content, additions include the complete incorporation of the previously separate arrayMap data collection [5, 6] and of datasets from external resources and projects such as The Cancer Genome Atlas (TCGA; 7, 8]) or cBioPortal [9], as well as the recurrent collection and re-processing of array-based data from NCBI's GEO or EMBL-EBI's ArrayExpress [10, 11]. Additionally, data content updates have followed the previous methodology of publication-based data extraction where feasible. Beyond the data expansion, a tight integration with projects of the Global Alliance for Genomics and Health (GA4GH [12]) and ELIXIR - such as serving for implementation-driven development of the Beacon API [13] - has led to an extension of 
the resource's features as well as adoption and promotion of emerging open data standards.

Here we present the latest updates on data content, structuring, standardization, access and other modifications made to the Progenetix resource.

\section{Data expansion and new features}

\section{Genomic profiling data}

Over the last two decades, thousands of cancer genomes studies have used the Gene Expression Omnibus (GEO; [14]) for deposition of data from array-based experiments. Data from GEO contributes a substantial fraction of the genomic screening data in the Progenetix collection and has again been expanded in both number of samples and represented platforms. Additionally, we systematically included suitable data from three more resources: ArrayExpress [15], cBioPortal (cBP) [16] and The Cancer Genome Atlas (TCGA) 17. project. As in the previous database updates, we have also included data directly derived from publication supplements and from collaborative projects. Table 1 shows statistics of samples within each resource. Table 2 reports the overall data growth and sample counts stratified by cancer loci since the last update. [4.

Table 1. Statistics of samples from various data resources

\begin{tabular}{cccccc}
\hline Data Source & GEO & ArrayExpress & cBioPortal & TCGA & Total \\
\hline No. Studies & 898 & 51 & 38 & 33 & $1^{\prime} 939$ \\
\hline No. samples & $63^{\prime} 568$ & $4^{\prime} 351$ & $19^{\prime} 712$ & $22^{\prime} 142$ & $138^{\prime} 663$ \\
Tumor & $52^{\prime} 090$ & $3^{\prime} 887$ & $19^{\prime} 712$ & $11^{\prime} 090$ & $15^{\prime} 357$ \\
Normal & $1^{\prime} 478$ & 464 & 0 & $11^{\prime} 052$ & $23^{\prime} 306$ \\
\hline Classifications & & & & & \\
ICD-O (Topography) & 100 & 54 & 88 & 157 & 209 \\
ICD-O (Morphology) & 246 & 908 & 265 & 140 & 491 \\
NCIt & 346 & 148 & 422 & 182 & 788 \\
\hline Collections & & & & & \\
Individuals & $63^{\prime} 568$ & $4^{\prime} 351$ & $19^{\prime} 712$ & $10^{\prime} 995$ & $127^{\prime} 549$ \\
Biosamples & $63^{\prime} 568$ & $4^{\prime} 351$ & $19^{\prime} 712$ & $22^{\prime} 142$ & $138^{\prime} 663$ \\
Callsets & $63^{\prime} 568$ & $4^{\prime} 351$ & $19^{\prime} 712$ & $22^{\prime} 376$ & $138^{\prime} 930$ \\
Variants & $5^{\prime} 514^{\prime} 126$ & $118^{\prime} 4170$ & $1^{\prime} 778^{\prime} 096$ & $2^{\prime} 654^{\prime} 065$ & $10^{\prime} 716^{\prime} 093$ \\
\hline
\end{tabular}

The "ArrayExpress Archive of Functional Genomics Data", hosted by European Molecular Biology Laboratory - European Bioinformatics Institute (EMBL-EBI), stores functional genomics data submitted by research groups and projects. In this update, we 
Table 2. Data growth by cancer loci

\begin{tabular}{lcc} 
Cancer loci & No. in 2014 & No. in 2021 \\
\hline Hematopoietic and reticuloendothelial systems & 5269 & 18482 \\
Lymph nodes & 2345 & 5988 \\
Breast & 2271 & 15790 \\
Cerebellum & 1439 & 3465 \\
Brain, NOS & 1342 & 6608 \\
Cerebrum & 1201 & 1712 \\
Liver & 1180 & 3237 \\
Stomach & 1155 & 3176 \\
Skin & 1073 & 3343 \\
Connective, subcutaneous and other soft tissues & 1058 & 2526 \\
Kidney & 1018 & 3617 \\
Colon & 1001 & 5182 \\
Ovary & 733 & 3963 \\
Prostate gland & 735 & 4485 \\
Lung and bronchus & 699 & 10321 \\
Nervous system, NOS & 667 & 926 \\
Urinary bladder & 587 & 1961 \\
Cervix uteri & 529 & 1331 \\
Peripheral nerves incl. autonomous & 523 & 1479 \\
Esophagus & 454 & 1890 \\
Pancreas & 426 & 1620 \\
Thyroid gland & 404 & 1260 \\
Heart, mediastinum, and pleura & 383 & 771 \\
Bones, joints and articular cartilage & 350 & 1205 \\
Spleen & 278 & 636 \\
Other & 4522 & 16268 \\
Total & 31642 & 115359 \\
& & \\
& &
\end{tabular}

have incorporated the cancer-related genomic profiles which do not have corresponding GEO entries using our analysis pipeline. Overall, data from ArrayExpress added 3'887 samples from 44 projects which resolve to 143 distinct cancer types according to NCIt. Similar to the GEO data acquisition procedure, we have used a combination of text mining methods and expert curation for annotation of technical metadata and biomedical parameter.

The "cBioPortal for Cancer Genomics" is an open-access resource for cancer genomics data, representing different types of molecular screening data from 19'712 samples, derived from 38 studies and mappable to 422 NCIt cancer types. The largest part of genomic data is based on whole exome sequencing analyses from the MSK-TARGET [18] pipeline, with CNA data accessed directly as segment files in genome version hg19/GRCh37. The data was converted into GRCh38 with the segment-liftover tool [19], and oncology classifications as well as relevant clinical data 
were incorporated into our database.

The Cancer Genome Atlas project provides a set of multi-omics data with extensive structured meta-data annotation for a large collection of cancer types, currently through NCBI's Genomic Data Commons Data Portal (https://portal.gdc.cancer.gov). In this update, we incorporated its CNV profiling data as well as transformed the relevant clinical information into our system (Figure 1).

\section{Data processing update}

Genomic profiling data in Progenetix originates from a large number of studies which are based on different molecular-cytogenetic and sequencing based technologies. In order to maximize qualitative homogeneity of the final CNA calls, we prefer to download source files with the least amount of pre-processing and apply our in-house data processing pipeline from the arrayMap project [5]. Currently, our analysis workflow handles the raw data based processing for 13 Affymetrix SNP array platforms, including 9 genome-wide arrays - 10K (GPL2641),50K (Hind240 and Xba240)

(GPL2004 and GPL2005), 250K (Nsp and Sty) (GPL3718 and GPL3720), Genome-wide SNP (5.0 and 6.0) (GPL6894 and GPL6801, respectively), CytoScan (750K and HD) (GPL18637 and GPL16131) arrays (GPL-prefixed platform coding in brackets according to GEO standard) - as well as the 4 cancer-specific "Oncoscan" arrays - GPL18602, GPL13270, GPL15793 and GPL21558.

\section{Allele-specific copy number variation}

For the subset of SNP array based experiments - where the status of both alleles can be evaluated separately - we have analyzed allele-specific copy number data (ASCN) and incorparated $35^{\prime} 897 \mathrm{LOH}$ profiles into the database. ASCN potentiates new analysis on the same samples. First, probe-wise it gives an overview of germline variant landscape, as used in determining the ancestry background. Second, it allows detection of loss of heterozygosity $(\mathrm{LOH})$ events, including of copy-number neutral event (CN-LOH) which e.g. can be commonly observed in haematological malignancies due to a selective process for duplication of minor disease-prone germline alleles [20, 21]. Lastly, it acts as a second reference for CNA to combat the variability caused by known wave artefacts 
from array technologies [22]. For all SNP arrays, we have implemented a pipeline to determine probe-wise B-allele frequency (BAF) of SNP probes and perform subsequent segmentation [23, 24]. Subsequently, we use ASCN to assess ancestry provenance of the samples 25] and store the LOH regions of the samples in our genomic variants database.
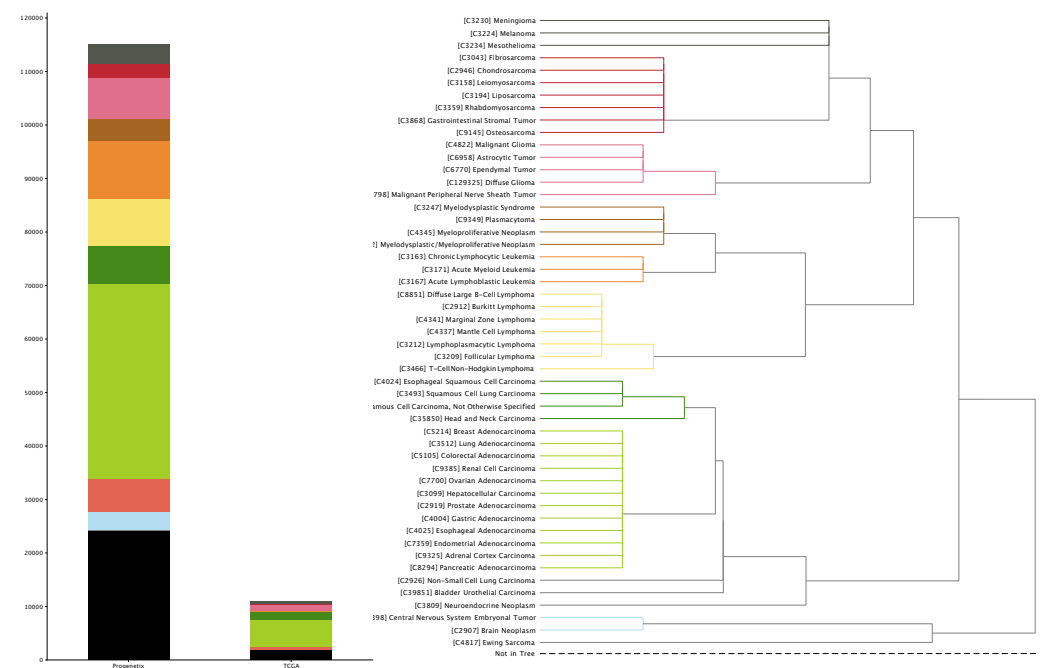

Fig 1. The currently available CNA data points in Progenetix and TCGA Progenetix database contains 115'357 cancer samples with 92'307 mapped to the 51 defined critical nodes in NCIt ontology tree and 23'050 samples not mapped to the tree (black); whereas TCGA repository contains 11'090 samples with 9'103 samples mapped and 1987 sample not mapped to the tree (black). Colors of the stacked barplot (left) match the branch colors on NCIt ontology tree (right)

\section{Meta-data updates}

Over the last years, a number of initiatives have addressed the lack of - or inconsistencies in - data standards for the representation and exchange of technical and "bio"-metadata in life sciences. Since 2016, the Progenetix resource has served as test bed for the implementation-driven design of metadata schemas and data exchange protocols, in conjunction with GA4GH workstreams and driver projects. For the structure and content of the Progenetix resource, this involvement led to the forward-looking adoption of hierarchical ontology classes as a core concept for a robust annotation of categorical metadata such as 'biocharacteristics' (e.g. phenotypes, disease categories) as well as the use of registered identifiers for external references using a CURIE syntax. Importantly, the resource informed the development and implemented the principles of a robust hierarchical data model for genomic data collections and exchange formats. 


\section{NCIt ontology mapping}

Since its establishment, Progenetix has made use of the International Classification of Diseases in Oncology, 3rd Edition (ICD-O 3) [26] for cancer sample classification. While the combination of the ICD-O Morphology and Topography coding systems depicts diagnostic entities with high specificity, the current ICD-O is limited in its representation of hierarchical concepts and does not easily translate to modern ontologies. In comparison, the National Cancer Institute Thesaurus (NCIt; access through http://bioportal.bioontology.org/ontologies/NCIT) is a dynamically developed hierarchical ontology which empowers layered data aggregation and transfer between classification systems and resources. However, due to the comparatively recent development and ongoing expansions, NCIt terms are rarely used in primary sample annotations. In the recent Progenetix update, we performed a data-driven generation of ICD-O - NCIt mappings and added the derived NCIt codes to all (existing and new) samples, to take advantage of NCIt's hierarchical structure for data retrieval, analysis and exchange (Figure $4 \mathrm{~B}$ ).

\section{Data summary based on the NCIt hierarchy tree}

All cancer samples in Progenetix have been annotated with an NCIt code, resulting in currently 789 distinct NCIt terms. However, as the definition of increasingly specific NCIt terms outruns their incorporation into the hierarchical tree, 98 of these terms so far are not represented in the tree hierarchy. For better illustration, we define 51 prominent nodes under which we summarize and visualize the data collection, where the disease types are both conceptually distinctive (e.g. "carcinoma" as category is too broad and thus its child nodes will be used) and include a considerable number of samples under the term or its child terms. This brings about additional 324 (60 in TCGA) terms not mappable to the selected nodes, resulting in 23,050 (1987 for TCGA) samples excluded from the summary tree counts (black bar in left panel of Figure 1). For terms with multiple occurrences in the tree we define the preferred path to the selected node by prioritizing morphology-based separation. The sample collection in Progenetix as compared to TCGA is summarized with reference to the NCIt coding system (Figure 1, Supplementary Table 1). 


\section{CNV data content by cancer type}

With cancer genomes grouped in the 51 NCIt nodes, we assessed their differences in the CNV landscape. The fraction of genome with a copy number alteration (CNV fraction) varies widely among the cancer types (Figure 2 Supplementary Figure 1). Among the most studied cancer types, the breast carcinoma shows a consistent CNV profile as an earlier analysis with frequent chr1q,8q,16p,17q,20 gain and 8p,16q,17p,18,22q loss [27]; the CNV pattern in cervical (chr3 gain), colorectal(chr7, 8q, 13, and 20q gain and 8p, $17 \mathrm{p}$, and 18 loss) carcinoma also correspond with previous observation [28]; similarly for T-cell non-Hodgkin lymphoma [29] and myelodysplastic syndrome [30. In addition, the genome-wide $\mathrm{LOH}$ profile also shows distinction among the cancer types in evaluation (42 out of 51 with at least 20 samples; Supplementary Figure 2). LOH profile of a cancer genome complements its CNV profile with the information of allelic loss. We highlight here a few prominent patterns which have been previously reported: chr3p and 9 in esophageal squamous cell carcinoma [31, 32]; chr18q in colorectal carcinoma [33]; chr13q, 16q and 17p in hepatocellular carcinoma 34].

\section{Uberon anatomy ontology}

While the ICD-O topography system provides organ and substructure specific mapping rooted in traditional clinical and diagnostic aspects of a "tumor entity", UBERON is a cross-species anatomical structural ontology system closely aligned with developmental processes 35. Its relationship structure allows integrative queries linking multiple databases (e.g. Gene Ontology [36], Protein Ontology [37]), description logic query within the same organism (linking related organs) and between model animals and humans. In this resource update, we have mapped all existing ICD-O T codes to UBERON terms, and additionally have provided those as part of the Monarch initiative 38, with our latest mapping table made available through a Github repository progenetix/icdot2uberon).

\section{Provenance by geography}

As part of the curated metadata provided in the sample representation, we have included geographic point coordinates for each individual sample. As this information is 


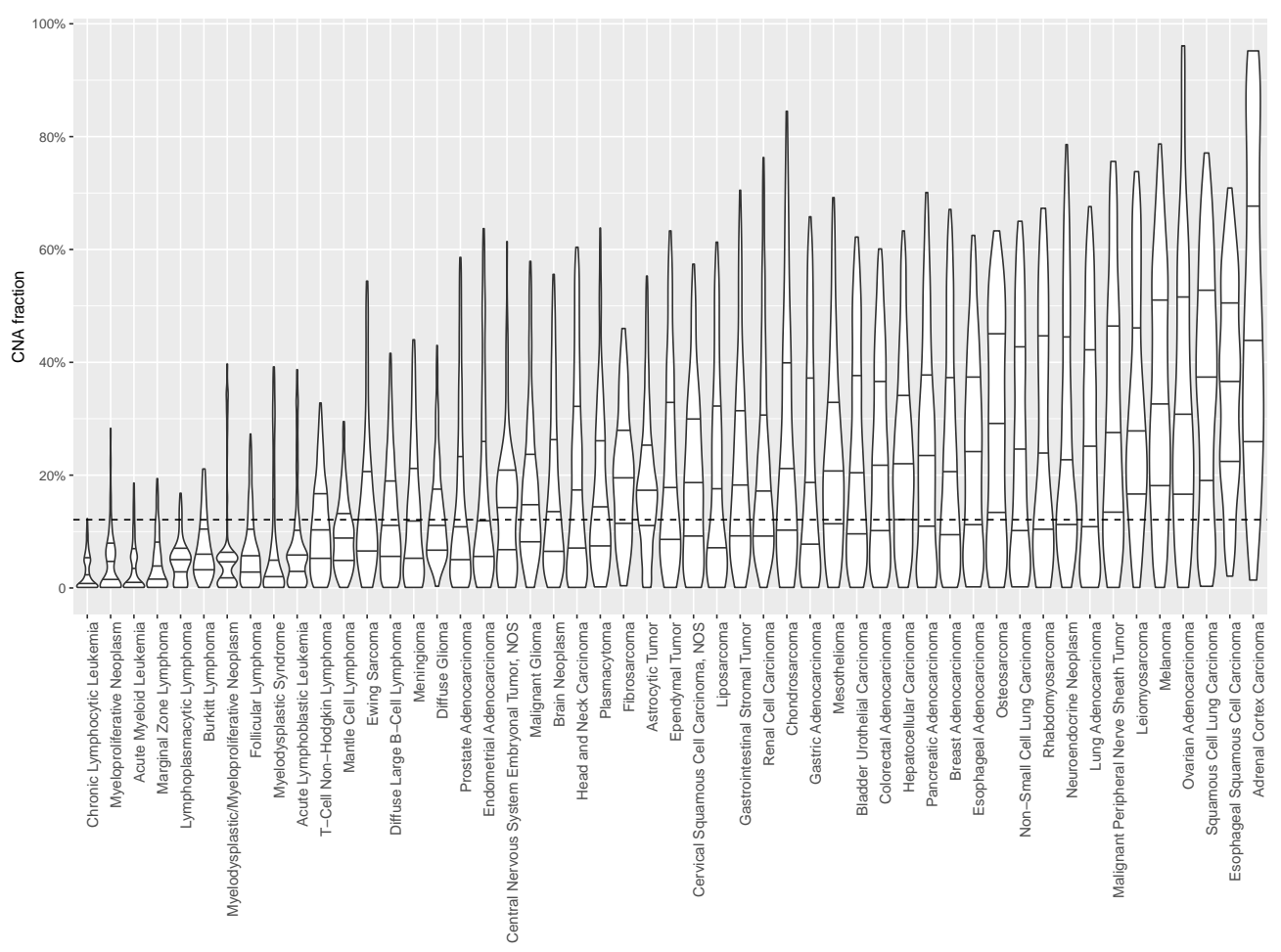

Fig 2. Distribution of genomic CNV fraction in 51 NCIt umbrella nodes illustrated with violin plot, showing median, first and third quantile. Dashed line indicates the global median CNV fraction at $12.1 \%$. Each category contains between 104 and 11804 CNV profiles (median 904; See Supplementary Table 1).

often missing from individual sample annotations, we previously have applied a mapping procedure to assign the samples' approximate geographic origins. For samples with the submitter's contact available from repository entries a default point-location in the corresponding city was used; otherwise that of the corresponding author of the associated publication was used. Associated publications were also explored for more detailed descriptions of sample origin. Point coordinates for each city were obtained using the external geographic database GeoNames (www.geonames.org), as detailed previously [39].

\section{Provenance by ancestry group}

While providing a good approximation for the geographic origin of cancer profiling data, which can e.g. be useful for epistemic validation and decision processes, the geographic location of the studies provides limited specificity regarding individual sample provenance, especially when assessing correlations between genomic variants and 
ancestral population background. Beyond the scope of high-penetrance variants like mutations in the BRCA1/2 [40, 41], or RB genes [42] in cancer predisposition, other studies have asserted an influence of genetic background on tumor development 43, 44, 45, 46]. Previously we have developed a method for deriving ancestry groups from un-masked germline variants in cancer genomes, based on reference populations studied in the 1000 Genomes Project [25]. For samples in Progenetix with accessible SNP data, population groups were assigned based on the reference categories mapped to Human Ancestry Ontology (HANCESTRO) terms (Supplementary Table 2). Where available, the respective data is now represented under the "populations provenance" schema for the corresponding biosample entries.

\section{Updated data access modalities}

Since the last release, we have adopted the GA4GH data schema standards and migrated to Phenopackets 47-formatted response delivery with modified data access points in the user interface. Information about application programming interface (API) methods are provided through the documentation pages

(https://info.progenetix.org/categories/API).

\section{Data standards}

In many genomic repositories, databases are structured around experimental outcomes (e.g. variants from a DNA sequencing experiments as collections of VCF files). Recent attempts in evaluating sensible meta-schemas for the representation of genomic variants and related biological or technical metadata, especially with respect to empowering data federation over flexible, networked resources, have led to a set of emerging meta-models and data schemas 48. The data storage and representation models for the Progenetix resource have been designed to comply with concepts developed by the previous GA4GH Data Working Group [12, 49] and subsequent GA4GH work streams, documented e.g. by the SchemaBlocks $\{\mathrm{S}\}$ 
initiative (http://schemablocks.org). One of the core concepts is the "individual biosample(s) - variants" meta-model which is applicable to cancer-related analyses with potentially multiple samples representing different stages in the course of disease as well as the underlying genomic background. This hierarchical model provides a solid representation and connection between the physical source of the data and the logical genotyping information and adapts to various scenarios for data aggregation and analysis.

\section{User interface}

The completely re-designed user interface provides flexibility and versatility in query parameters and types and optimized the response delivery. Technically, the query interface for retrieval of sample specific data is built on top of a forward-looking implementation of the GA4GH Beacon API [13] with features from the upcoming version 2 of this standard.

Figure 3 shows the current web interface to perform a CNA query with start and end position range with filter options for cancer type, tissue location, morphology, cell line or geographic location. The top panel of the result page shows a summary with the number of matched samples, variants, calls and the frequency of alleles containing the CNA (Figure 3E). The "Phenopackets" link returns a json document of biosamples with the phenopacket-formated response. The "UCSC region" links externally to a UCSC browser track providing an overview of the genomic elements which map to the region of the observed variants. Also, customized visualization is enabled in the linked page "visualization options", e.g. for selected chromosomal regions and grouping by subsets or studies. The lower panel is organized in four sections. 1) the "Result" tab (Figure $3 \mathrm{~F}$ ) shows the genome-wide CNA by the percentage of samples with yellow $(+)$ as $\mathrm{CN}$ gain and blue (-) as CN loss. Below the CNA plot is a table showing the list of subsets as defined by ICD-O-3 and NCIt Ontology terms sorted by frequency of matched samples within that subset. 2) the "Biosamples" tab (Figure $3 \mathrm{G}$ ) shows information of matched biosamples, i.e. description, classifications and external identifiers. The table can be downloaded as json or csv format. The further detail of the biosample can be accessed by clicking the biosample id. 3) The "Biosamples Map" tab (Figure 3H) shows 
a world map with the matched geological locations highlighted. 4) the "Variants" tab (Figure 35) shows the variant "digest" (concatenated format with chromosome, start, end position and type of the CNA) and its corresponding biosample and callset.

Likewise, the table can be downloaded as json or csv format.

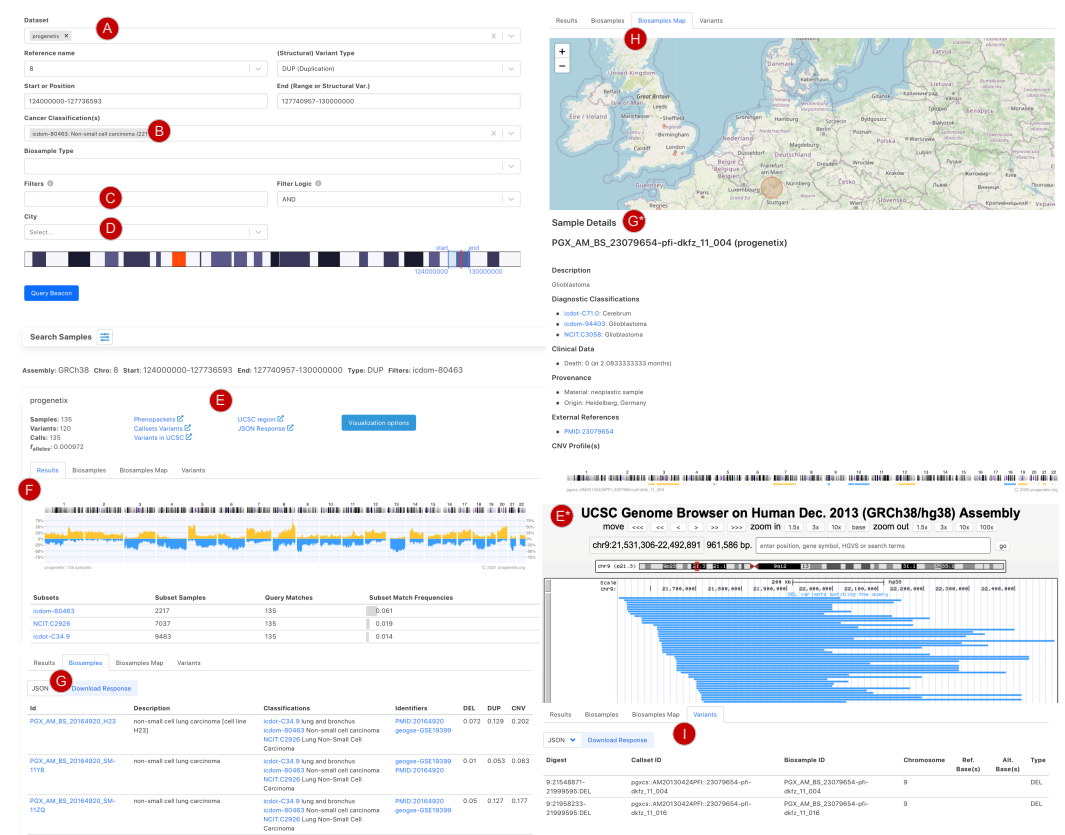

Fig 3. Beacon-style query using fuzzy ranges to identify biosamples with variants matching the CNA range This example queries for a continuous, focal duplicaton covering the complete MYC gene's coding region with $<=6 \mathrm{Mbp}$ in size. A: Filter for dataset. B: filter for cancer classification (NCIt and ICD-O-3 ontology terms available), C: additional filter, e.g. cellosaurus D: additional filter for geographic location. E: external link to UCSC browser to view the alignment of matched variants; F: cancer type classification sorted by frequency of the matched biosamples present in the subset; G: list of matched biosamples with description, statistics and reference. More detailed biosample information can be viewed through $i d$ link to the sample detail page. H: matched variants with reference to biosamples can be downloaded as json or csv format.

Figure 4 shows the additional functional interfaces and services provided by the Progenetix project. Users can search for publications or studies by publication title, author names or the geographic location of the research center. Then, navigation extends to the summary of publications with the number of samples catalogued by technology and availability in database as well as options to visualize the associated samples (Figure 4A). Users can also access samples from the NCIt hierarchical tree or other classification systems (e.g. ICD-O, UBERON) to select a subset of cancer types for summary statistics and visualization (Figure $4 \mathrm{~B}$ ). Alternatively, users can also 
upload their own data for single or multiple samples to visualize genome-wide CNA

(Figure $4 \mathrm{C})$.

A

Progenetix Publication Collection

The current page lists articles describing whole genome screening (WGS, WES, acGH, cCGH) experiments in cancer, registered in the Progenentix publication collection. For each

publication the table indicates the numbers of samples analysed with a

Search

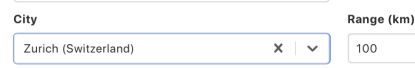

Publications (37) Samples

id Publication eCOH acCH WES wos pgx am

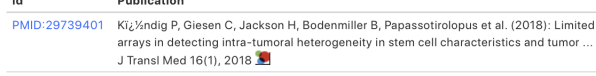

PMID:29556019 Pillonel V, Juskevicius D, Ng CKY, Bodmer A, Zettl le tal. (2018): High-throughput sequencing of nodal marginal zone lymphomas identifies recurrent BRAF mutations. Leukemia, 2018

PMID: 27658049 Riba J, Renz N, Niemölller C, Bleul S, Pfeifer D, Stosch JM, Metzeler KH, Hackanson B, Lübbert M, Duyster J, Koltay $0 \begin{array}{llllll}3 & 0 & 0 & 3 & 0\end{array}$ P, Zengente R, Cliaus R, Zimmermann S, Becker H.. Cells Isolated via Single-Cell Printing
plos one

PMID: 27491809

Eusco N, Geyer FC, De Filippo MR, Martelotto LG, Ng CK, Piscuogglio S, Guerin--Rocco E, Schultheis AM, Fuhrmann L, Wang L, Jungbluth AA, Burke KA, Lim RS, Vincent-Salomon A, Bamba M, Moritani S, Badve SS, Ichinara S, Ellis
IO, Reis-Filho JS, Weigett B., (2016): Genetic events in the progression of adenoid cystic carcinoma of the breast to

high-grade...
Modern pathology : an official journal of the United States and Canadian Academy of Pathology, Inc

PMID:26632267 Kovac M, Blattmann C, Ribi S, Smida J, Mueller NS, Engert F, Castro-Giner F, Weischenfeldt J, Kovacova M, Krieg A,

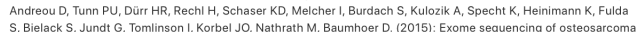
reveals mutation signatures reminiscent of BRCA deficienecy...
Nature communications

B Cancer Types
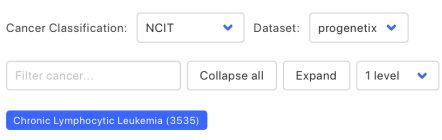

$\checkmark$ NCIT:C3262: Neoplasm (111840 samples)

> NCIT:C3263: Neoplasm by Site (106563 samples)

$\checkmark$ NCIT:C4741: Neoplasm by Morphology (106398 samples)

$\checkmark$ NCIT:C27134: Hematopoietic and Lymphoid Cell Neoplasm (24961 samples)

$\checkmark$ NCIT:C3161: Leukemia (11451 samples)

ᄀ NCIT:C3172: Myeloid Loukemia (5635 samples)

$\checkmark$ NCIT:C3483: Chronic Leukemia (3622 samples)

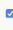

NCIT:C3163: Chronic Lymphocytic Leukemia (3535 samples)

C Data visualization Upload

File format

Data has to be submitted as tab-delimited .tsu segment files. An example file is being provided here.

Fig 4. Demonstration of further functionality pages: A. Publication search; B. NCIT hierarchical tree navigation A: Cancer genomics-associated publications are recorded with number of samples stratified by technology used. The publications can be filtered by keywords; B: Part of the sample subsets contained in Progenetix under the hierarchical NCIt classification tree. It allows for selection of sample subsets at different levels; C: User can upload custom segment files for data visualization. 


\section{Other improvements}

\section{Genome version update}

All samples have been updated to Genome Reference Consortium Human Build 38 (GRCh38). The process has been completed in a step-wise manner. Preferably, for samples with available probe-specific array data, either GRCh38 mapped platform data files were used for re-processing of the original files; alternatively, a lift-over of the probe data and subsequent re-segmentation was performed. For those cases where only called CNA data had been collected we applied our recently published "segment-liftover" tool [19] for the efficient re-mapping of continuous segments. Overall, more than $99.99 \%$ of probes and more than $99 \%$ of segments could be recovered successfully.

\section{Cell line collection}

Cancer cell lines are important models for understanding the molecular mechanisms of malignant diseases and have a prominent role in pharmacological screening procedures. Besides the primary tumor data, the Progenetix data collection also includes genomic profiling experiments using in vitro models. Recently, we introduced a systematic update of cell line annotations based on Cellosaurus, a comprehensive knowledge resource on cell line data with extensive annotations and mappings to a variety of classifications and ontologies [50]. We meticulously assigned Cellosaurus IDs for the cancer cell line samples as well as the ICD-O morphology and topography codes based on the NCIt term annotated by Cellosaurus. At this time, Progenetix includes a total of 5764 samples corresponding to 2162 different cancer cell lines, representing 259 different cancer types (NCIt). While so far we provide the option to search for cell lines by applying a "cellosaurus" filter either in the web interface (e.g. "cellosaurus:CVCL_0030" for HeLa cell line samples) or in the API query, work on a dedicated cell line data access tool is under way.

\section{Conclusion}

The Progenetix resource provides an extensive collection of oncogenomic data with a focus on individual genome-wide CNA profiles and the use of modern ontologies and 
data schemas to render curated biological and technical metadata, as well as thorough references to external repositories and annotation resources. Through aggregation of data from thousands of individual research studies as well as several consortium derived collections, to our knowledge Progenetix database currently constitutes the largest public, freely accessible resource for pre-computed CNA profiles and associated phenotypic information and additional metadata dedicated to cancer studies. While the application of uniform genomic data formats and a benchmarked data processing pipeline minimizes biases from separate studies, the forward-looking implementation of emerging ontology standards facilitates the integrative and comparative analysis across a vast range of cancer types. The tight integration with GA4GH product development and standardization processes guarantees the compatibility with emerging data federation approaches and widest re-utilization of the resource's data. For the future, besides the continuous maintainance and expansion of the existing data types, we will work towards enhancing clinical and diagnostic annotation, expanding cross-database references and the types of genomic variant data as well as active data sharing and integration through networked services and platforms.

\section{Supplementary Information}

Supplementary Figure 1: The genome-wide CNV landscape of samples in the 51 NCIt categories. For each category, we randomly sampled around 150 genome profiling data and plotted the percentage of CN gain or loss across the genome. Yellow $(+)$ indicates copy gain and Blue (-) indicates copy loss. The genomic profiles were ordered with hierarchical clustering with Euclidean distance and median linkage. NOS: not otherwise specified.

Supplementary Figure 2: The genome-wide LOH landscape of samples in the 51 NCIt categories. For each category, we randomly sampled around 100 genome-wide $\mathrm{LOH}$ profiles and plotted the percentage of samples harboring $\mathrm{LOH}$ in regions across the genome in the (-) direction, as an indication for uniparental allelic loss. The genomic profiles were ordered with hierarchical clustering with Euclidean distance and median linkage. NOS: not otherwise specified.

Supplementary Table 1: The number of samples belonging to the 51 NCIt 
summary terms in Progenetix.

Supplementary Table 2: The mapping table between the 1000 Genomes reference population labels and HANCESTRO ontology terms.

\section{Acknowledgments}

Work on the Progenetix Beacon implementation was supported by ELIXIR and through the BioMedIT Network project of SIB Swiss Institute of Bioinformatics in the realm of the Swiss Personalized Health Network. We'd like to thank Amos Bairoch for support with the cell line annotations. Improvements in data annotation concepts were highly influenced through the GA4GH community. 


\section{References}

[1] Hanahan, D., Weinberg, R. (2011). Hallmarks of cancer: the next generation. Cell, 144(5), $646-674$.

[2] Albertson, D. G., Collins, C., McCormick, F., Gray, J. W. (2003). Chromosome aberrations in solid tumors. Nature genetics, 34(4), 369-376.

[3] Baudis, M., Cleary, M. (2001). Progenetix.net: an online repository for molecular cytogenetic aberration data. Bioinformatics, 17(12), 1228-1229.

[4] Cai, H., Kumar, N., Ai, N., et al. (2014a). Progenetix: 12 years of oncogenomic data curation. Nucleic Acids Res, 42(Database issue), D1055-62.

[5] Cai, H., Kumar, N., Baudis, M. (2012). arraymap: a reference resource for genomic copy number imbalances in human malignancies. PLoS One, 7(5), e36944.

[6] Cai, H., Kumar, N., Bagheri, H., et al. (2014b). Chromothripsis-like patterns are recurring but heterogeneously distributed features in a survey of 22,347 cancer genome screens. $B M C$ Genomics, 15(1), 82 .

[7] Cancer Genome Atlas Research Network, Weinstein, J., Collisson, E., et al. (2013). The cancer genome atlas pan-cancer analysis project. Nat Genet, 45(10), 1113-1120.

[8] National Cancer Institute (2013). The Cancer Genome Atlas Program. https: //www.cancer.gov/about-nci/organization/ccg/research/structural-genomics/tcga. Accessed: 2021-01-26.

[9] Gao, J., Aksoy, B., Dogrusoz, U., et al. (2013). Integrative analysis of complex cancer genomics and clinical profiles using the cbioportal. Sci Signal, 6(269), pl1.

[10] National Center for Biotechnology Information (NCBI) (2002). Gene Expression Omnibus. Www.ncbi.nlm.nih.gov/geo/ Accessed: 2021-01-25.

[11] The European Bioinformatics Institute (EMBL-EBI) (2003). Arrayexpress. Www.ebi.ac.uk/microarray-as/aer/ Accessed: 2021-01-26.

[12] Global Alliance for Genomics and Health (2016). Genomics. a federated ecosystem for sharing genomic, clinical data. Science, 352(6291), 1278-1280.

[13] Fiume, M., Cupak, M., Keenan, S., et al. (2019). Federated discovery and sharing of genomic data using beacons. Nat Biotechnol, 37(3), 220-224. 
[14] Edgar, R., Domrachev, M., Lash, A. (2002). Gene expression omnibus: Ncbi gene expression and hybridization array data repository. Nucleic Acids Res, 30(1), 207-210.

[15] Athar, A., Füllgrabe, A., George, N., et al. (2019). Arrayexpress update-from bulk to single-cell expression data. Nucleic acids research, 47(D1), D711-D715.

[16] Cerami, E., Gao, J., Dogrusoz, U., et al. (2012). The cbio cancer genomics portal: an open platform for exploring multidimensional cancer genomics data. Cancer Discov, 2(5), 401-404.

[17] Network, C. G. A. R. (2008). Comprehensive genomic characterization defines human glioblastoma genes and core pathways. Nature, 455(7216), 1061-1068.

[18] Cheng, D. T., Mitchell, T. N., Zehir, A., et al. (2015). Memorial sloan kettering-integrated mutation profiling of actionable cancer targets (msk-impact): a hybridization capture-based next-generation sequencing clinical assay for solid tumor molecular oncology. The Journal of molecular diagnostics, 17(3), 251-264.

[19] Gao, B., Huang, Q., Baudis, M. (2018). segment_liftover : a python tool to convert segments between genome assemblies [version 1; referees: awaiting peer review]. F1000Research, $\mathbf{7}(319)$.

[20] O’Keefe, C., McDevitt, M. A., Maciejewski, J. P. (2010). Copy neutral loss of heterozygosity: a novel chromosomal lesion in myeloid malignancies. Blood, 115(14), $2731-2739$.

[21] Mullighan, C. G., Goorha, S., Radtke, I., et al. (2007). Genome-wide analysis of genetic alterations in acute lymphoblastic leukaemia. Nature, 446(7137), 758-764.

[22] Ai, N., Cai, H., Solovan, C., Baudis, M. (2016). Cnara: reliability assessment for genomic copy number profiles. BMC Genomics, 17(1), 799.

[23] Ortiz-Estevez, M., Bengtsson, H., Rubio, A. (2010). ACNE: a summarization method to estimate allele-specific copy numbers for affymetrix snp arrays. Bioinformatics, 26(15), $1827-1833$.

[24] Olshen, A. B., Venkatraman, E., Lucito, R., Wigler, M. (2004). Circular binary segmentation for the analysis of array-based dna copy number data. Biostatistics, $\mathbf{5}(4)$, $557-572$.

[25] Huang, Q., Baudis, M. (2020). Enabling population assignment from cancer genomes with snp2pop. Scientific reports, 10(1), 1-9. 
[26] World Health Organization and others (2013). International classification of diseases for oncology ( icd-o)-3rd edition, 1st revision.

[27] Cai, H., Gupta, S., Rath, P., et al. (2015). arraymap 2014: an updated cancer genome resource. Nucleic acids research, 43(D1), D825-D830.

[28] Ried, T., Hu, Y., Difilippantonio, M. J., et al. (2012). The consequences of chromosomal aneuploidy on the transcriptome of cancer cells. Biochimica et Biophysica Acta (BBA)-Gene Regulatory Mechanisms, 1819(7), 784-793.

[29] da Silva Almeida, A. C., Abate, F., Khiabanian, H., et al. (2015). The mutational landscape of cutaneous t cell lymphoma and sezary syndrome. Nature genetics, 47(12), 1465.

[30] Xu, L., Gu, Z.-H., Li, Y., et al. (2014). Genomic landscape of cd34+ hematopoietic cells in myelodysplastic syndrome and gene mutation profiles as prognostic markers. Proceedings of the National Academy of Sciences, 111(23), 8589-8594.

[31] Tarmin, L., Yin, J., Zhou, X., et al. (1994). Frequent loss of heterozygosity on chromosome 9 in adenocarcinoma and squamous cell carcinoma of the esophagus. Cancer research, 54(23), 6094-6096.

[32] Kuroki, T., Trapasso, F., Yendamuri, S., et al. (2003). Allele loss and promoter hypermethylation of vhl, $\operatorname{rar}-\beta$, rassf1a, and fhit tumor suppressor genes on chromosome $3 p$ in esophageal squamous cell carcinoma. Cancer research, 63(13), 3724-3728.

[33] Armaghany, T., Wilson, J. D., Chu, Q., Mills, G. (2012). Genetic alterations in colorectal cancer. Gastrointestinal cancer research: GCR, 5(1), 19.

[34] Nishida, N., Fukuda, Y., Kokuryu, H., et al. (1992). Accumulation of allelic loss on arms of chromosomes 13q, 16q and 17p in the advanced stages of human hepatocellular carcinoma. International journal of cancer, 51(6), 862-868.

[35] Mungall, C. J., Torniai, C., Gkoutos, G. V., et al. (2012). Uberon, an integrative multi-species anatomy ontology. Genome biology, 13(1), R5.

[36] Gene Ontology Consortium (2008). The gene ontology project in 2008. Nucleic acids research, 36(suppl_1), D440-D444.

[37] Natale, D. A., Arighi, C. N., Barker, W. C., et al. (2007). Framework for a protein ontology. In BMC bioinformatics, volume 8, page S1. Springer. 
[38] Mungall, C. J., McMurry, J. A., Köhler, S., et al. (2017). The monarch initiative: an integrative data and analytic platform connecting phenotypes to genotypes across species. Nucleic acids research, 45(D1), D712-D722.

[39] Carrio-Cordo, P., Baudis, M. (2018). Mountains and chasms: Surveying the oncogenomic publication landscape. Oncology, pages 1-12.

[40] Miki, Y., Swensen, J., Shattuck-Eidens, D., et al. (1994). A strong candidate for the breast and ovarian cancer susceptibility gene brca1. Science, 266(5182), 66-71.

[41] Wooster, R., Bignell, G., Lancaster, J., et al. (1995). Identification of the breast cancer susceptibility gene brca2. Nature, 378(6559), 789-792.

[42] Friend, S. H., Bernards, R., Rogelj, S., et al. (1986). A human dna segment with properties of the gene that predisposes to retinoblastoma and osteosarcoma. Nature, 323(6089), 643-646.

[43] Amundadottir, L. T., Sulem, P., Gudmundsson, J., et al. (2006). A common variant associated with prostate cancer in european and african populations. Nature genetics, $\mathbf{3 8}(6)$, 652.

[44] Stacey, S. N., Manolescu, A., Sulem, P., et al. (2007). Common variants on chromosomes 2q35 and 16q12 confer susceptibility to estrogen receptor-positive breast cancer. Nature genetics, 39(7), 865 .

[45] Tenesa, A., Farrington, S. M., Prendergast, J. G., et al. (2008). Genome-wide association scan identifies a colorectal cancer susceptibility locus on 11q23 and replicates risk loci at 8q24 and 18q21. Nature genetics, 40(5), 631.

[46] Wu, C., Hu, Z., Yu, D., et al. (2009). Genetic variants on chromosome 15q25 associated with lung cancer risk in chinese populations. Cancer research, 69(12), 5065-5072.

[47] Julius OB Jacobsen, Peter N Robinson, C. J. M. (2019). Phenopackets schema. https://phenopackets-schema.readthedocs.io/en/latest/ Accessed: 2021-01-25.

[48] Wagner, A. H., Babb, L., Alterovitz, G., et al. (2021). The ga4gh variation representation specification (vrs): a computational framework for the precise representation and federated identification of molecular variation. bioRxiv.

[49] Lawler, M., Siu, L., Rehm, H., et al. (2015). All the world's a stage: Facilitating discovery science and improved cancer care through the global alliance for genomics and health.

Cancer Discov, 5(11), 1133-1136. 
bioRxiv preprint doi: https://doi. org/10.1101/2021.02.15.428237; this version posted February 16,2021 . The copyright holder for this preprint (which was not certified by peer review) is the author/funder, who has granted bioRxiv a license to display the preprint in perpetuity. It is made available under aCC-BY 4.0 International license.

[50] Bairoch, A. (2018). The cellosaurus, a cell-line knowledge resource. Journal of biomolecular techniques: JBT, 29(2), 25. 